\title{
Fractal structure, power-law distribution and hadron spectrum
}

\section{Airton Deppman*}

Instituto de Física da Universidade de São Paulo

E-mail: deppmaneusp.br

Properties of hadron structure and of multiparticle production in high energy collisions are described by a fractal structure of strong interacting systems which is derived from the scaling properties of QCD or, in general, of Yang-Mills field theory. It is shown that those systems are described by the Tsallis non extensive statistics, and the entropic index, $q$, is determined by the filed theory parameters. Experimental evidences are discussed.

XIII Quark Confinement and the Hadron Spectrum - Confinement2018

31 July - 6 August 2018

Maynooth University, Ireland

${ }^{*}$ Speaker. 


\section{Introduction}

Experiments of high energy collisions have accumulated lots of data, allowing for a deep investigation of the quark-gluon plasma (QGP) properties. Information on quarks and gluons propagation in the medium can be investigated with high accuracy, and the properties of the bulk matter can give information on confinement and on the Equation of States of the quark-gluon plasma and of hadronic matter, opening the opportunity to use that information in other related fields, as in the composition and stability of stars, or in the study of the primordial universe.

Hagedorn[1,2] with the self-consistent thermodynamics predicted, aside the well known limiting temperature, or Hagedorn temperature, the exponential behavior of energy and momentum distributions, as well as the exponential increase of the hadron mass spectrum. The success of his theory triggered the development of hadron resonance gas models[3] and was the basis for the proposal of the quark-gluon plasma[4]. However, as the energy of the experiments increased and a large region of momentum distributions was measured, it was found that Hagedorn's theory was not able to describe completely the experimental results. With the increasing importance of QCD, Hagedorn's theory became obsolete, but more recently it was shown that the power law behavior could be explained by using Tsallis distribution [5]. With the present work we intend to show that it is possible to reconcile the thermodynamical self-consistency idea proposed by Hagedorn with gauge field theory in general and particularly with QCD. We will show that renormalized fields can arrange themselves in a fractal structure which can be described by non extensive statistics, and discuss that an extended version of Hagedorn's theory can contribute to our understanding of the high energy collisions experiments and of QCD. The generalization of Hagedorn theory by adopting Tsallis statistics was performed in Ref. [6] and the demonstration that a fractal structure can explain the emergence of Tsallis statictics was done in Ref. [7].

\section{Fractal structure and non extensive statistics}

The emergence of Tsallis statistics in strong interacting systems has been shown to be related to a fractal structure of thermodynamical functions [7], which were called thermofractals. These systems feature the following properties: the have internal structure with $N^{\prime}$ components, each of them being thermofractals; the kinetic energy $K$ and the internal energy, $E$, of the constituent particles are such that the ratio $E / K$ vary according to a distribution $\tilde{P}(E / K)$. It is possible to show that, starting from the classical Boltzmann statistics, one finally finds that the best way to describe the thermodynamical properties of thermofractals is by using Tsallis statistics [5].

The probability for the total energy of a thermofractal to be between $\mathrm{U}$ and $\mathrm{U}+\mathrm{dU}$ is, therefore, given by [7]

$$
P(U) d U=\lambda^{-D} \sum_{\left\{N^{\prime}\right\}} \prod_{i} A k T\left[\tilde{P}\left(m_{i}\right)\right]^{v} e^{-\beta U_{i}} d \mu_{i} d^{3} \pi
$$

where A is a normalization constant, $\lambda=T / T_{o}$, with $T_{o}$ being some reference temperature, and $\pi=p / k T$ and $\mu=m / k T$ are normalized momentum and mass. The new variable, $\varepsilon$, is defined by $\varepsilon_{i} / k T=E_{i} / K$, with $U_{i}=m_{i}+K_{i}, E_{i}$ being the particle total energy and $K_{i}$ its kinetic energy. We observe that the effective energy, $\varepsilon$, allows to scale the internal parton energy with the global scaling instead of the kinetic energy. With the introduction of these variables A is dimensionless. 
The equation above establishes a relation between the energy distribution of the initial system, and the internal energy distribution of its components.

A crucial point is to notice that, being both thermofractals, the probability distributions $P(U)$ for the initial system and the probability distribution $\tilde{P}\left(m_{i}\right)$ must be the same. Therefore one has to find a function $P(\varepsilon)$ that, substituted in Eq. (2.1) in the places where $P(U)$ and $\tilde{P}\left(m_{i}\right)$ appear, provides a solution to that equality. Such function was found in Ref. [7], and can be written in the form

$$
\tilde{P}(\varepsilon)=A\left[1+(q-1)\left(\frac{\varepsilon}{k \tau}\right)\right]^{-\frac{1}{q-1}}
$$

where

$$
q-1=\frac{2}{3 N}(1-v)
$$

and

$$
\tau=(q-1) N T .
$$

The right hand side of Eq.( 2.2) can be recognized as the Tsallis distribution, which arises from application of the non extensive statistics proposed by tsallis [5]. The parameter $q$ is known as entropic index, and $\tau$ is an effective temperature.

The connection between Tsallis and Boltzmann statistics provided by the introduction of thermofractal structure allows a deeper understanding on how the non extensive statistics generalizes the extensive statistics, and affords the determination of the entropic index in terms of the parameters of the fractal structure, as given by Eq. (2.3). In addition, it is shown that the temperature, $T$, in Boltzmann statistics, differs from the temperature, $\tau$, in Tsallis statistics, determining a relation between the two parameters, as shown in Eq. (2.4).

Observe that $N$ and $v$ are parameters of the fractal structure, with $N$ being the number of components in the internal structure of the thermofractal. As $N$ increases, the energy of each component decreases, in order to conserve the total energy. As $N \rightarrow \infty$, the internal energy of each component becomes negligible, therefore also the effects of its internal structure. In this case the system behaves as an extensive ideal gas, and in fact we observe in Eq. (2.3) that in such limit we have $q \rightarrow 1$, exactly the limit when Boltzmann statistics is recovered from Tsallis statistics [5].

The parameter $v$ regulates the relative importance of the internal structure to the total energy fluctuation. Indeed, if $v=1$, small probabilities are less important, and the energy fluctuation is dominated by the most probable configurations of the thermofractal. Small variations of the internal energy are unimportant, and the system behaves as an ideal gas. Again, from Eq. (2.3), we see that in such case $q=1$ and Boltzmann statistics is restored. As $v$ decreases, small probabilities becomes more relevant and fluctuations of internal energy of the components are more important. In the case $v \rightarrow 0$, the effects of several levels of internal structure are relevant to describe the energy fluctuation of the system. For $v=1$ a self-similar solution is not possible [7]. We see that Boltzmann statistics is recovered when $N \rightarrow \infty$.

A detailed analysis of the thermofractal structure was performed in Ref. [8], where it was shown that the system presents a fractal structure in the energy-momentum space. The scaling properties of thermofractals were further investigated in terms of renormalization group equation in Ref. $[9,10]$. 
Scaling properties are present in many systems and processes [11], and are in many cases related to complex structures, fractal dimensions and self-organization. Since QCD is a special case of Yang-Mills field theory, which is renormalizable, it is interesting to investigate if fractal structures is allowed in this field theory.

\section{Yang-Mills fields and fractal structure}

The simplest scale free gauge field theory has Lagrangian density including bosons and fermions given by

$$
\mathscr{L}=-\frac{1}{4} F_{\mu \nu}^{a} F^{a \mu \nu}+i \bar{\psi} \gamma_{\mu} D_{i j}^{\mu} \psi_{j}
$$

where $F_{\mu \nu}^{\alpha}=\partial_{\mu} A_{v}^{a}-\partial_{v} A_{\mu}^{a}+g f^{a b c} A_{\mu}^{b} A_{v}^{c}$ and $D_{i j}^{\mu}=\partial_{\mu} \delta_{i j}-i g A^{\alpha \mu} T_{i j}^{a}$, with $\psi$ and $A$ being, respectively, the fermion and the vector fields, and $f^{a b c}$ being the structure constants of the group and $T^{a}$ the matrices of the group generators in the fermion representation

One important aspect of Yang-Mills field theory is that it is renormalizable, that is, when regularization is used to avoid infinities, the original vertex functions are related to new vertex functions with renormalized parameters, $m$ and $\bar{g}$, as

$$
\Gamma(p, m, g)=\lambda^{-D} \Gamma(p, \bar{m}, \bar{g})
$$

This property is mathematically described by the renormalization group equation [12, 13, 14, 15]. Such equation is known as Callan-Symanzik equation $[16,17,18]$, and is given by

$$
\left[M \frac{\partial}{\partial M}+\beta_{g} \frac{\partial}{\partial \bar{g}}+\gamma\right] \Gamma=0
$$

where $M$ is the scale parameter, and the $\beta$-functions are defined as

$$
\beta_{g}=M \frac{\partial \bar{g}}{\partial M}
$$

$D=D_{o}+d$, with $D_{o}$ being the natural dimension of the phase-space. In general, $d$ is not necessarily an integer, therefore the scaling dimension, $D$, may be fractionary. The parameter $\gamma$ in the equation indicates the anomalous dimension of the fields under scaling, and is given by a combination of the scaling dimensions of the fields $\psi$ and $A$.

The partition function of a quantum system can be written as

$$
Z=\operatorname{Tr}<\psi|U(\mathrm{i} \beta H, 0)| \psi>
$$

where the trace is taken over all possible gas configuration.

$H$ is the complete Hamiltonian for the interacting systems, but in the Schwinger perturbative approach it is usual to write the amplitude in terms of the free-fields Hamiltonian, $H_{o}$, and in terms of the coupling constant, $g$, resulting in

$$
<\varphi|U(\mathrm{i} \beta H, 0)| \varphi>=<\varphi\left|U\left(\mathrm{i} \beta H_{o}, 0\right)\right| \varphi>+<\varphi\left|g U\left(\mathrm{i} \beta H_{o}, 0\right)\right| \varphi>+\ldots .
$$


In the one-particle irreducible representation, those states can be written as

$$
\left|\varphi(n)>=\mathscr{S} \prod_{i=1}^{N(n)}\right| \varphi\left(m_{i}, p_{i}\right)>
$$

where $\mid \varphi\left(m_{i}, p_{i}\right)>$ represents the state of a particle with mass $m_{i}$ and momentum $p_{i}$, and is an autovector of the Hamiltonian $H_{o, i}$ such that $H_{o}=H_{o, 1}+H_{o, 2}+\ldots$. The states $\mid \varphi\left(m_{i}, p_{i}\right)>$ correspond to parton states with self-energy calculated up to order $n$, and we will refer to these states as effective partons. $\mathscr{S}$ represents the (anti)simmetrization operator.

The perturbative approach given by Eq. (3.6), is represented by diagrams like those shown in Fig. 1. The scale invariance means that, after proper scaling, the loop in a higher order graph is identical to a loop in lower orders. This is a fundamental consequence of the Callan-Symanzik equation, and it is of great importance in what follows.

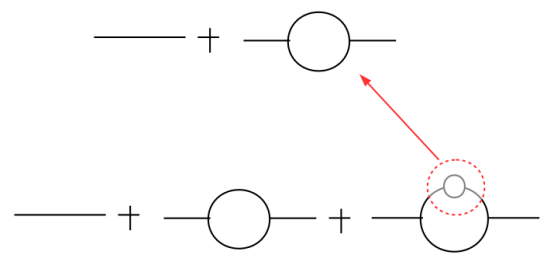

Figure 1: Scaling relation between diagrams at different orders. Red dashed lined indicates that the loop at higher order is equivalent to a loop at a lower order if proper normalization is used.

The number of effective partons at the nth order of perturbative calculation depends on the configuration, and by $N^{\prime}$ we will refer to the set of configurations with $N^{\prime}$ partons. As the order of perturbative calculation, $n$, increases, the number of partons $N^{\prime}$ tends to increase, but not linearly. With larger numbers of partons present, high order configurations present partons with smaller energies, and therefore with smaller fluctuations of their internal energies.

Summation of all graphs at different orders leads to the summation of configurations with different number of particles. Describing the system in Fokker space allows to sum over the different number of particles, and Wick rotation gives rise to the Boltzmann factor, hence we obtain the partition function of the gas ${ }^{1}$

$$
Z=\lambda^{-D} \sum_{\left\{N^{\prime}\right\}} \prod_{i=1}^{N^{\prime}} \int d m_{i}\left[\tilde{P}\left(m_{i}\right)\right]^{v} d^{3} p<\varphi_{i, m}\left|e^{-\beta H_{o, i}}\right| \varphi_{i, m}>,
$$

where we include a distribution of the internal energy, or effective mass, of the generated partons, $\tilde{P}(m), H_{o, i}$ is the Hamiltonian operator for a free particle. Here, $\left\{N^{\prime}\right\}$ indicates that the summation is over all possible configurations of $N^{\prime}$ particles gas, $\lambda$ is a scaling factor, and $v$ is a parameter that will be determined below. With ortonormalized states, one gets

$$
Z=\lambda^{-D} \sum_{\left\{N^{\prime}\right\}} \prod_{i} \int d m_{i}\left[\tilde{P}\left(m_{i}\right)\right]^{v} e^{-\beta U_{i}} d^{3} p
$$

\footnotetext{
${ }^{1}$ For simplicity, we will use $m$ for indicating the effective mass, instead of using the symbol $\bar{m}$.
} 
The relativistic energy can be written as $U_{i}=m_{i}+p_{i}^{2} /\left(2 m_{i}\right)+O\left(p^{4} / m^{3}\right)$ and in this case, Equation 2.1 can be written in terms of $m$ and $K$ as

$$
P(U) d U=\lambda^{-D} \sum_{\left\{N^{\prime}\right\}} \prod_{i}\left(\frac{F}{k T}\right)^{3 N / 2-1} e^{-\alpha F / k T} d\left(\frac{K}{k T}\right) A k T\left[\tilde{P}\left(m_{i}\right)\right]^{v} \frac{d \mu}{F},
$$

where

$$
F=\frac{p_{i}^{2}}{2 m_{i}}
$$

and

$$
\alpha=1+\frac{m_{i}}{F_{i}}+O\left(p_{i}^{2} / m_{i}^{2}\right)
$$

Of course we have

$$
U_{i}=\alpha F_{i}
$$

For future convenience, we introduce the variable $\varepsilon$ such that

$$
\frac{\varepsilon_{i}}{k T}=\frac{m_{i}}{F_{i}}+O\left(p_{i}^{2} / m_{i}^{2}\right)
$$

Since $m$ and $p$ are independent, also $\varepsilon$ and $K$ are independent, we can integrate the equation above on the kinetic energy, $K$, resulting

$$
P(U) d U=\lambda^{-D} \sum_{\left\{N^{\prime}\right\}} A k T \prod_{i} \Gamma(3 N / 2)\left(1+\frac{\varepsilon}{k T}\right)^{3 N / 2}[\tilde{P}(\varepsilon)]^{v} \frac{d \varepsilon}{K},
$$

where we made use of the relation $d \varepsilon=d \mu / K$. Notice that, due to scale invariance, the parent parton represented by the initial line in the graph must have the same properties as any other parton in the system, after proper scaling. Therefore $\mathrm{P}(\mathrm{U})$, which is the fluctuation of the internal energy, can be understood as the distribution of the parton mass, thus

$$
P(U) \sim P(\varepsilon)
$$

that is, there is self-similarity in the gauge field.

Relation (3.16) shows that the internal energy, $U$ of the parent parton fluctuates according to the same distribution that the internal energy of its constituents do. The internal energy is identified with the parton effective energy.

These results are similar to those obtained for thermofractals by following a completely different approach [7, 8], where Eqs. (3.10) and (3.15), as well as the self-similarity relation (3.16). This result shows that if Tsallis statistics is used in thermodynamical approaches to hadronic physics all details of the complex structure are naturally incorporated in the thermodynamical relations derived from the entropic form proposed for such statistics [5], what facilitates the calculations. In what follows, $1 / k T$ will be considered our scaling parameter, with the same meaning as $M$ in the Callan-Symanzik equation.

According to relation (3.16), the properties of a parton at any scale are similar to the properties of the partons at another scale, and the distribution of the internal energy at the parent parton, after proper rescaling, is equal to the distribution of internal energy, or effective mass, of its components. 
Since the energy and momentum of the partons are defined at the vertex, it is natural to suppose that the effective constant at each vertex is related to the distribution of mass of the compounding partons. Thus we use the following ansatz for the effective coupling:

$$
\bar{g}=\prod_{i=1}^{N^{\prime}}\left[1+(q-1) \frac{\varepsilon_{i}}{k \tau}\right]^{-\frac{v}{q-1}},
$$

with $N^{\prime}$, here, referring to the number of branches in the first order expansion, what depends on the field topology. For quark and gluon fields, for instance, $N^{\prime}=2$, as well as for the field represented by the graphs in Figs 1.

It is interesting to note, at this point, the asymptotic behavior of the effective coupling proposed here. This can be done by considering an initial scale, $T_{o}$, where the coupling is determined, that is, $\bar{g}\left(T_{o}\right)=g$, and the effective parton mass is $m$. Now we observe how the coupling constant behaves as we go to the next order by adding a loop in the graphical representation, as shown in Fig. 1. The variation of the effective coupling with respect to its initial value, $g$, at the initial scale is

$$
\delta \bar{g}=g^{N^{\prime}} \delta g
$$

with the factor $g^{N^{\prime}}$ coming from the field topology. From the definition of the coupling in Eq. (3.17), we have

$$
M \frac{\delta g}{\delta M}=\sum_{j=1} N^{\prime} \bar{g} M \varepsilon_{j}\left[1+(q-1) M \varepsilon_{j}\right]^{-1} .
$$

From here we can obtain the beta function, $\beta_{g}$, at the asymptotic limit, $M \rightarrow 0$ which, using Eqs. (3.18) and (3.19), results to be

$$
\beta_{g}=-\frac{N^{\prime} v}{q-1} g^{N^{\prime}+1}
$$

Hence the field theory is asymptotically free if $q>1$.

The asymptotic limit has been useful to understand many aspects of high energy collisions, and in particular to understand the asymptotic behavior under scale transformation. Here we can use this limit to understand the meaning of the parameter $q$ introduced in connection with the thermodynamical interpretation of the vertex function. From the Callan-Symanzik Eq. 3.3, we get, since the gauge theory satisfies such equation, that

$$
d-\frac{N^{\prime} v}{q-1}-\gamma=0
$$

where we have used the fact that the $\Gamma$ function scales with a dimension $D=D_{o}+d$ and that the fields and operators in the vertex function scales with $D_{o}+\gamma$. From here we obtain the relation between the parameter $q$, which in Tsallis statistics is the entropic index, in terms of parameters that are intrinsic to the gauge field structure. In the case of QCD, where $\psi$ refers to quark fields and $A$ refers to gluon fields, we have $N^{\prime}=2$, therefore

$$
\frac{2 v}{q-1}=d-\gamma
$$


The parameters $d$ and $\gamma$ are related to the intrinsic features of the fields and of the particular $\Gamma$ function analyzed. The anomalous dimensions for the vector and fermion fields may be different, and they contribute proportionally to the number of external lines corresponding to each field, so

$$
\gamma=n \gamma_{A}+n^{\prime} \gamma_{\psi}
$$

A quantitative comparison must show that the value for $q$ found in experimental data analysis corresponds to the value determined by Eq. (3.29). To this end, we write Eq. (3.5) in terms of the field propagators, $G_{f}$, with $f=A$ or $f=\psi$ for the vector and fermion propagators, respectively. With this, we obtain

$$
Z=\sum_{\left\{N^{\prime}\right\}} \int(2 \pi)^{4 N^{\prime}} k_{i}<\varphi\left|\prod_{f=1} N^{\prime} G_{f} \bar{g}\right| \varphi>,
$$

where $k_{i}=p_{i} /(2 \pi)$. First order approximation it will lead to[14]

$$
Z=Z_{o}\left[1+\frac{1}{16 \pi^{2}} g^{2} \gamma \ln \left(\frac{-p^{2}}{M^{2}}\right)\right]
$$

what leads to

$$
\delta Z=Z-Z_{o}=\left[\frac{1}{16 \pi^{2}} g^{2} \gamma \ln \left(\frac{-p^{2}}{M^{2}}\right)\right] Z_{o} .
$$

It follows from here and from Callan-Symanzik equation that

$$
\frac{2 v}{q-1}=d-\left(n \gamma_{\psi}+n^{\prime} \gamma_{A}\right)
$$

where the amplitude anomalous dimension, $d$, is such that [12]

$$
d-\left(n \gamma_{\psi}+n^{\prime} \gamma_{A}\right)=\left[\frac{11}{3} c_{1}-\frac{4}{3} c_{2}\right] g^{2}
$$

Therefore, we find

$$
\frac{2 v}{q-1}=\left[\frac{11}{3} c_{1}-\frac{4}{3} c_{2}\right]
$$

where

$$
\left\{\begin{array}{l}
c_{1} \delta_{a b}=f_{a c d} f_{b c d} \\
c_{2} \delta_{a} b=t r T_{a} T_{b},
\end{array}\right.
$$

therefore relating the entropic index, $q$, to fundamental parameters of the field theory.

Such fractal structure implies Tsallis statistics, and gives a phenomenological motivation to the use of non extensive statistics in high energy collisions, and explains why Hagedorn's theory must be modified to accommodate non extensivity. In fact, the non extensive self-consistent thermodynamics [6] predicts power-law distributions for energy and momentum that are in good agreement with experimental data $[19,20,21]$, and gives a hadron mass spectrum formula which can describe the mass distribution of the known hadrons, starting from pion mass [20]. All these results are accomplished with a parameter $q$ which is approximately independent of the collision energy and of the particle species, with $q=1,14 \pm 0.01$. Qualitatively, this finding is in agreement with the conclusion we arrived in the present work, with the parameter $q$ found to be related to constant 
parameters that are related to the structure of the field theory. Now we proceed to a quantitative comparison between the value of $q$ found from the parameters of QCD, as described in Eq. (3.29), and the value obtained from experimental data analyses.

Quantitatively, the parameters $c_{1}$ and $c_{2}$ are related to the number of colors and flavors by $c_{1}=N_{c}$ and $c_{2}=N_{f} / 2$. Using $N_{c}=N_{f} / 2=3$ we get

$$
\frac{11}{3} c_{1}-\frac{4}{3} c_{2}=9,
$$

which, using Eq. (3.29), leads to $q=1.11$, while from experimental data analysis it results $q=$ $1.14 \pm 0.01$, showing a fair agreement between theory and experiments. It must be noticed that the analyses of $p p$ collision data are performed assuming that there are no other effects on the outcome of measured transversal momentum, such as elliptic flow, however today it is clear that even in such small systems this effect is present, and can interfere on the value of $q$ obtained through $p_{T^{-}}$ distribution analysis. In fact, from the study of hadron mass spectrum [20] the value for $q$ is in better agreement with the theoretical one.

The results obtained here gives a solid basis in terms of QCD for the thermodynamical approach used many times to describe high energy data. Also, it explains the self-similarity that has been observed by different means in the outcomes of high energy collisions [22, 23, 24] and the behavior of hadron mas spectrum [20]. Applications of such phenomenological approaches have been used to calculate the equation of states for neutron stars [25], to evaluate the phase-transition line at finite chemical potential [26] and to understand the hadron structure [27]. All those applications can now find support from QCD theory.

\section{Conclusions}

In conclusion, we have shown in the present work that renormalizable field theories leads to fractal structures, which can be studied, from a thermodynamical point of view, with Tsallis statistics. A recursive method allows to perform non perturbative calculations to describe the particles structure governed by the gauge theory. In the case of multiparticle production, the calculations lead to a thermodynamical description where non extensive statistics must be used. The results obtained here give a solid basis from QCD to the use of non extensive self-consistent thermodynamics to describe properties of strong interacting systems and to the use of thermofractal structure to describe hadrons.

\section{References}

[1] R. Hagedorn, CERN - TH. 520 65/166/5 (1965).

[2] R. Hagedorn, Nuovo Cimento Suppl. 3 (1965) 147.

[3] R. Venugopalan and M. Prakash, Nucl. Phys. A546 (1992) 718.

[4] N. Cabibbo and G. Parisi, Phys. Lett. 59B (1975) 67.

[5] C. Tsallis, J. Stat. Phys. 52 (1988) 479.

[6] A. Deppman, Physica A 391 (2012) 6380. 
[7] A. Deppman, Phys. Rev. D 93 (2016) 054001.

[8] A. Deppman, T. Frederico, E. Megias, D. P Menezes, Entropy 20 (2018) 633.

[9] A. Deppman, Universe 3 (2017) 62.

[10] A. Deppman, Adv. Theor. Phys. (2018) 9141249.

[11] G. West, in "Scale, The universal laws of life, growth, and death in organisms, cities and companies", Penguin Book, NY 2018.

[12] H. D. Politzer, Phys. Reports 14 (1974) 129.

[13] H. Georgi and H. D. Politzer, Phys. Rev. D9 (1974) 416.

[14] D. Gross and F. Wilczek, Phys. Reports 14 (1974) ??

[15] D. Gross and F. Wilczek, Phys. Rev. D9 (1974) 980.

[16] C. G. Callan, Jr., Phys. Rev. D 2 (1970) 1541.

[17] K. Symanzik, Commun. math. Phys. 18 (1970) 227.

[18] K. Symanzik, Commun. math. Phys. 23 (1971) 49.

[19] J. Phys. G: Nucl. Part. Phys. 39, 095004 (2012) 98.

[20] L. Marques, E. Andrade-II and A. Deppman, Phys. Rev. D 87 (2013) 114022.

[21] L. Marques, J. Cleymans and A. Deppman, Phys. Rev. D 91 (2015) 054025.

[22] M. Tokarev and I. Zborovsky, EPJ Web Conf. 141 (2017) 02006.

[23] I. Zborovsky and M.V. Tokarev, Phys. Rev. D75, (2007) 094008.

[24] G. Wilk and Z. Włodarczyk, Phys. Lett. B (2013) 163.

[25] D.P. Menezes, A. Deppman, E. MegÄśías and L.B. Castro, Eur. Phys. J. A 51 (2015) 155.

[26] E. Megías, D. P. Menezes, and A. Deppman, Physica A 421, 15 (2015).

[27] P.H.G. Cardoso, T.N. da Silva, A. Deppman, D.P. Menezes, Eur. Phys. Journal A 53(2017) 191. 